\title{
Healthcare provider relational quality is associated with better self-management and less treatment burden in people with multiple chronic conditions
}

This article was published in the following Dove Press journal:

Patient Preference and Adherence

26 September 2017

Number of times this article has been viewed

\author{
David T Eton ${ }^{1,2}$ \\ Jennifer L Ridgeway ${ }^{1,2}$ \\ Mark Linzer ${ }^{3}$ \\ Deborah H Boehm 4 \\ Elizabeth A Rogers ${ }^{5}$ \\ Kathleen J Yost ${ }^{1,2}$ \\ Lila J Finney Rutten ${ }^{1,2}$ \\ Jennifer L St Sauver ${ }^{1,2}$ \\ Sara Poplau ${ }^{4}$ \\ Roger T Anderson ${ }^{6}$ \\ 'Department of Health Sciences \\ Research, ${ }^{2}$ Robert $D$ and Patricia \\ E Kern Center for the Science of \\ Health Care Delivery, Mayo Clinic, \\ Rochester, MN, ${ }^{3}$ Division of General \\ Internal Medicine, Hennepin County \\ Medical Center, ${ }^{4}$ Minneapolis Medical \\ Research Foundation, ${ }^{5}$ Division of \\ General Internal Medicine, University \\ of Minnesota Medical School, \\ Minneapolis, MN, ${ }^{6}$ Department of \\ Public Health Sciences, University \\ of Virginia School of Medicine, \\ Charlottesville, VA, USA
}

Correspondence: David T Eton Division of Health Care Policy \& Research, Department of Health Sciences Research, Mayo Clinic, 200 First Street Southwest, Rochester, MN 55905, USA

Tel + I 507293 I353

Fax + I 507284 |73।

Email eton.david@mayo.edu
Purpose: Having multiple chronic conditions (MCCs) can lead to appreciable treatment and self-management burden. Healthcare provider relational quality (HPRQ) - the communicative and interpersonal skill of the provider - may mitigate treatment burden and promote selfmanagement. The objectives of this study were to 1) identify the associations between HPRQ, treatment burden, and psychosocial outcomes in adults with MCCs, and 2) determine if certain indicators of HPRQ are more strongly associated than others with these outcomes.

Patients and methods: This is a cross-sectional survey study of 332 people with MCCs. Patients completed a 7-item measure of HPRQ and measures of treatment and self-management burden, chronic condition distress, self-efficacy, provider satisfaction, medication adherence, and physical and mental health. Associations between HPRQ, treatment burden, and psychosocial outcomes were determined using correlational analyses and independent samples $t$-tests, which were repeated in item-level analyses to explore which indicators of HPRQ were most strongly associated with the outcomes.

Results: Most respondents (69\%) were diagnosed with $\geq 3$ chronic conditions. Better HPRQ was found to be associated with less treatment and self-management burden and better psychosocial outcomes $(P<0.001)$, even after controlling for physical and mental health. Those reporting $100 \%$ adherence to prescribed medications had higher HPRQ scores than those reporting less than perfect adherence $(P<0.001)$. HPRQ items showing the strongest associations with outcomes were "my healthcare provider spends enough time with me", "my healthcare provider listens carefully to me", and "I have trust in my healthcare provider".

Conclusion: Good communication and interpersonal skills of healthcare providers may lessen feelings of treatment burden and empower patients to feel confident in their self-management. Patient trust in the provider is an important element of HPRQ. Educating healthcare providers about the importance of interpersonal and relational skills could lead to more patient-centered care.

Keywords: patient-provider relationship, multi-morbidity, adherence, patient-centered care, trust

\section{Introduction}

Many of today's chronic illnesses require continuous self-management. The ability to self-manage is critical and has been closely tied to physical and mental health outcomes across a range of chronic illnesses. ${ }^{1}$ Self-management requirements are often magnified in patients coping with multiple chronic conditions (MCCs). In populations with MCCs, increasing difficulty with performing self-management tasks has been linked to declines in physical and mental health. ${ }^{2}$ Moreover, patient perception of 
treatment and self-management burden, that is, the perceived "workload" of health care and its impact on functioning and well-being, has been associated with greater distress, lower self-efficacy, and poorer adherence to prescribed medications. ${ }^{3}$ Identifying factors that may relieve treatment and self-management burden and promote self-management capabilities could facilitate patients' engagement with their care and improve outcomes.

In a recent qualitative study, we found that a number of factors may lessen feelings of treatment and self-management burden in patients with MCCs. ${ }^{4}$ Prominent among these were positive and constructive elements of the patient-provider relationship, including good provider communication and listening skills. Prior studies have shown that better provider communication is associated with better patient selfmanagement and adherence to medical regimens, ${ }^{5-9}$ with the relationship possibly being mediated by health self-efficacy. ${ }^{10}$ Conversely, perceptions of hurried communication (eg, doctors speaking too fast, using complex words, appearing distracted) have been related to poorer patient outcomes. ${ }^{11}$ Theoretical mechanisms of the impact of provider communication on patient health outcomes point to indirect effects, such that better patient-provider communication influences health outcomes through intermediate outcomes like improved adherence to medical regimens. ${ }^{12}$

Beyond communicative skill, studies also point to the importance of provider interpersonal skills, especially those that engender feelings of trust and respect from patients. "High-performing" physicians report establishing trust with their patients by maintaining eye contact, conveying empathy and understanding, leaning forward in their chair when speaking with patients, smiling, and not looking hurried. ${ }^{13}$ Feeling trust in one's physician has been linked to greater adherence to treatment recommendations, including medication adherence ${ }^{14,15}$ and engagement in healthy behaviors like exercise and smoking cessation. ${ }^{16}$ Chronically ill patients who feel greater trust and security in their relationships with providers may feel more empowered, confident, and motivated to manage the demands of their illness. ${ }^{17}$ Conversely, patients who perceive a lack of respect from their providers may develop negative attitudes about their prescribed care regimens. In a recent meta-synthesis of qualitative studies, Mohammed et al found that patients preferred to self-manage their medicines, sometimes with negative consequences, when they perceived that their providers were not giving them adequate time at appointments, were not paying enough attention to them, or seemed unwilling to consider their life circumstances. ${ }^{18}$ Proposed theoretical pathways support that a strong therapeutic alliance between healthcare professionals, patients, and caregivers (ie, a relationship that engenders trust and respect among all parties) can lead to better health outcomes indirectly by lowering patient distress, increasing satisfaction with providers, and enhancing patient commitment to treatment plans. ${ }^{12}$

\section{Study objectives}

An understanding of the quality of the patient-provider relationship requires attention to not only the provider's ability to communicate but also his/her interpersonal and relational skills, ${ }^{19}$ a distinction that was articulated some 30 years ago in seminal work by Hall et al. ${ }^{20}$ Comprehensive measures of communication and other aspects of the patient-provider relationship are available, such as the Interpersonal Processes of Care Survey, ${ }^{21}$ the Picker Institute Patient Experience Questionnaire, ${ }^{22}$ the Consumer Assessment of Healthcare Providers and Systems $\left(\mathrm{CAHPS}^{\circledR}\right)$ Clinician and Group Survey (CG-CAHPS), ${ }^{23}$ and the Trust in Physicians Survey. ${ }^{24}$ Such measures are typically used to assess the patient's perception of the relationship with one particular type of provider, the physician. However, patients with multi-morbidity seen in primary care clinics are often treated by healthcare teams that involve more than one type of healthcare provider. A brief measure of the patient-provider relationship that does not specify a provider type may offer a useful alternative for studies of patients with MCCs seen in primary care settings. With this backdrop, we formed 2 study objectives. First, we aimed to identify the associations between healthcare provider relational quality (HPRQ) - defined here as the communicative and interpersonal skill of the healthcare provider - and various self-management and psychosocial outcomes in adults with MCCs. To investigate these relationships, we employed a brief measure of HPRQ developed specifically for use in our studies of the treatment burden construct. ${ }^{3,4}$ The measure does not specify a provider type. Second, we were interested in determining if certain indicators of HPRQ are more strongly associated than others with self-management and psychosocial outcomes in adults with MCCs. This study represents a secondary analysis of data collected for a previously conducted study to validate a novel patient-reported measure of treatment burden. ${ }^{3}$

\section{Patients and methods}

\section{Sample}

Participants with MCCs were recruited from the Hennepin County Medical Center (Minneapolis, MN, USA) and the Mayo Clinic (Rochester, MN, USA) as part of a prior 
survey study to validate a novel patient-reported measure of treatment burden. ${ }^{3}$ Hennepin County Medical Center is Minnesota's largest public "safety-net" hospital providing care to many low-income, uninsured, and vulnerable persons. Mayo Clinic is a large, multispecialty, integrated practice located in southeast Minnesota. Patients were eligible if they 1) were $\geq 21$ years old, 2) were assigned to a primary care provider at either site, 3 ) had medical recordconfirmed diagnoses of $\geq 2$ chronic conditions (specifically conditions requiring self-management), ${ }^{25-28}$ and 4) had at least 1 medical record-confirmed encounter with a provider at either site within the past 18 months for their chronic conditions. Patients were ineligible if they had no documented research authorization per Minnesota state privacy law, Statute 144.335, 1997. Minnesota state law requires that healthcare providers obtain authorization to use health records for research from all patients who have received medical care after January 1, 1997. This includes consent to contact individuals for research. Patients lacking English language proficiency were excluded. A stratified random sample of 838 people was drawn from a list of eligible patients identified from the medical records of the 2 sites. Stratification factors included number of diagnosed conditions $(2,3$, or $4+)$, number of encounters with a provider in the past 18 months $(1-8,9-17,18+)$, and age $(<65$ or $\geq 65)$. The sample size was based on having adequate power for the psychometric analyses of the previously conducted treatment burden measure validation study ${ }^{3}$ with expectation of a minimum survey return rate of $40 \%$.

\section{Procedure}

A survey booklet consisting of study measures was prepared by the Mayo Clinic Survey Research Center and mailed to eligible patients along with a cover letter, a small gift to encourage participation (designer pen or $\$ 3$ gift card), and a postage-paid return envelope. To maximize returns, a second mailing to nonrespondents occurred 3 weeks after the first. The study was deemed exempt by both site Institutional Review Boards under category 2 of Title 45 Code of Federal Regulations (CFR) Part 46.101 (Protection of Human Subjects). A research study is eligible for category 2 exempt status if 1) it does not involve prisoners, 2) it does not involve an approved FDA-regulated product used in the course of medical practice, and 3 ) it is research that involves only the use of educational tests or survey procedures with (i) the information obtained recorded in such a manner that human subjects cannot be identified, either directly or through identifiers, and (ii) any disclosure of human subjects' responses outside the research would not reasonably place the subjects at risk of criminal or civil liability or be damaging to their financial standing, employability, or reputation. The research involved no procedures for which written consent is normally required outside of the research context (ie, the surveys); all criteria for waiver of consent documentation were met in accordance with 45 CFR 46.117, and hence, waiver of consent documentation was approved. The cover letter described the survey study, including how data would be aggregated for analysis and kept secure, and participants were told to skip any questions they did not want to answer. Participant anonymity was preserved in the following ways: 1) names and addresses were only recorded to create mailing address labels, 2) a unique study identifier was used to track survey returns and to link with medical record data, 3) returned survey data were pooled across all participants and only an aggregated dataset was analyzed (ie, no individual was identifiable in any analysis or report), and 4) the file containing participant names and addresses used to create mailing labels and link with medical record data was destroyed at the conclusion of the study. This report represents a secondary analysis of data collected for the treatment burden measure validation study. ${ }^{3}$ Several of the measures used were selected specifically to assess construct validity of the treatment burden measure developed in the parent study.

\section{Study measures}

\section{Healthcare provider relational quality}

Informed by a conceptual framework of factors that may lessen treatment burden in people self-managing MCCs, ${ }^{4}$ we created a brief, 7-item patient-reported measure of HPRQ. Items were written to operationalize specific patient experiences and issues as reflected in patient interviews and focus groups. ${ }^{4}$ These same interviews and focus groups were previously used to inform content for a novel measure of treatment burden. ${ }^{3}$ Some questions were adapted from items embedded within the Doctor Communication composite of the CG-CAHPS, ${ }^{23}$ and others were newly written. Items were reviewed by healthcare providers, health services researchers, and patient advocates and were cognitively pretested in patients with MCCs. ${ }^{3}$ In 2 rounds of cognitive interviews with 23 patients, all of the items of the HPRQ measure were found to be easy to understand and were rated as highly relevant and important aspects of health care. Details of the cognitive interviewing procedure are available in an online supplement to the study of Eton et al. ${ }^{3}$

The HPRQ measure defines a "healthcare provider" as "any doctor, nurse, or other health professional that helps 
treat you and provides you with medical care". Respondents are asked to think about their current health care and determine their agreement with the following statements: 1) My provider(s) listens carefully to me, 2) It is easy to communicate with my provider(s), 3) My provider(s) treats me with dignity and respect, 4) My provider(s) is up to date on my medical history, 5) My provider(s) spends enough time with me, 6) My provider(s) treats me as a whole person, and 7) I have trust in my provider(s). All items use a 4-point response scale ranging from strongly disagree to strongly agree. A raw score is computed by summing the item scores, imputing any unanswered items with the mean of the completed items as long as $>50 \%$ of the items (ie, 4 of 7 ) are answered. Imputing missing item scores in this manner has been used with other patient-reported measures. ${ }^{29}$ The raw score is transformed to a standardized 0-100 metric with a higher score indicative of better HPRQ.

\section{Treatment burden}

Three scales from the Patient Experience with Treatment and Self-management (PETS), a recently validated patientreported measure of perceived treatment burden, ${ }^{3}$ were used in this analysis. Scales included the 7-item Medical Information Burden scale, the 7-item Difficulty with Healthcare Services scale, and the 5-item Physical/Mental Exhaustion due to self-management scale. The items making up these scales are found in Table $\mathrm{S} 1$. Scale scores are standardized to a 0-100 metric with higher scores indicating greater treatment burden. The 3-item Convenience subscale of the Treatment Satisfaction Questionnaire for Medication (TSQM) ${ }^{30}$ was used to assess the ease and convenience of taking required medications for one's health conditions. Higher scores indicate greater perceived convenience. Both the PETS and TSQM are psychometrically sound and valid measures in patients with chronic health conditions..$^{3,30}$

\section{Distress, self-efficacy, satisfaction with provider, and medication adherence}

A 5-item Chronic Condition Distress Scale (CCDS) was adapted from the Diabetes Distress Scale $^{31}$ and specified generally for "health problems" (L Fisher, personal communication). The scale assesses a general feeling of distress associated with living with a chronic health condition (eg, feeling overwhelmed, feeling your friends or family are not providing you enough support, feeling your health condition keeps you from enjoying life). Wallston's 8-item Perceived Medical Condition Self-management (PMCSM) scale was used to assess self-efficacy or perceived competence in managing one's health condition. ${ }^{32}$ These measures have been shown to be reliable and valid in patients with chronic illnesses requiring self-management. ${ }^{31,32}$

To assess satisfaction with provider, an overall rating of healthcare providers was adapted from a numeric rating scale item of the CG-CAHPS survey. ${ }^{23}$ The item was tailored to fit this study (ie, "Overall, how would you rate your healthcare providers who treat you and provide you with medical care?") and used a 0-10 rating scale ranging from "worst healthcare providers possible" $(0)$ to "best healthcare providers possible" (10). A single-item self-report measure was used to assess adherence to physician-recommended medications ("always", "usually", or "sometimes"). Lower adherence has been shown to be associated with greater perceived treatment burden ${ }^{3}$ and worse control of blood pressure in patients with hypertension. ${ }^{14}$

\section{Demographics and covariates}

Demographic characteristics (age, race/ethnicity, and education), number of prescription medications, and overall physical and mental health were assessed using single items. Overall physical and mental health status was assessed using 2 items of the Patient-Reported Outcomes Measurement Information System (PROMIS) Global-10 measure. The PROMIS Global-10 provides global assessments of physical and mental health and has been shown to be valid and reliable in patients with chronic illnesses. ${ }^{33}$ The physical and mental health ratings are used as covariates in this study. Finally, gender, number and types of chronic conditions, and the number of recent encounters with a provider (in last 18 months) were extracted from the electronic medical record.

\section{Analyses}

Descriptive statistics (ie, means, medians, and frequency distributions) were used to describe survey respondents. Cronbach's alpha was used to check the internal consistency reliability of the HPRQ measure.

\section{Association of HPRQ measure and outcomes (study objective I)}

Pearson correlations $(r)$ were used to determine the extent that scores on the HPRQ measure are associated with patient distress, perceived treatment burden, medication convenience, self-management self-efficacy, and satisfaction with provider. We used Cohen's criterion benchmarks for small $(r=0.10)$, medium $(r=0.30)$, and large $(r=0.50)$ effect sizes to help interpret the relative size of the correlation magnitudes. ${ }^{34}$ 
Consistent with findings of prior studies and meta-analytic reviews, ${ }^{7,10,11,18,35}$ as well as proposed theoretical pathways linking patient-provider communication and relationships to health outcomes, ${ }^{12}$ we hypothesized that higher (better) HPRQ would be negatively correlated with distress (CCDS score) and perceived treatment burden (PETS scale scores) and positively correlated with medication convenience (TSQM score), self-efficacy (PMCSM score), and satisfaction with provider (healthcare provider rating). Further, we determined whether overall physical and mental health accounts for variance in these relationships by covarying the PROMIS physical and mental health item scores from the pairwise correlations (ie, partial correlation). If these factors are confounders, then the pairwise correlation magnitudes should be reduced and approach 0 once they are statistically controlled.

An independent samples $t$-test was used to determine whether scores on the HPRQ measure were associated with adherence to physician-prescribed medications. For this analysis, we created distinct groups by dichotomizing responses to the medication adherence question in the same manner as Schoenthaler et al (ie, "always take all my medications" vs "usually/sometimes take all my medications"). ${ }^{14}$ The magnitude of the group difference was determined using the effect size (Cohen's $d$ ) by dividing the difference of the group means by the pooled $\mathrm{SD}$, with $d=0.2,0.5$, and 0.8 considered small, medium, and large effect sizes, respectively. ${ }^{34}$ Consistent with findings of prior studies, reviews, and metaanalyses, ${ }^{5,6,8,14,15}$ and proposed theoretical mechanisms linking patient-provider communication and health outcomes, ${ }^{12}$ we hypothesized that greater adherence to medications would be associated with better HPRQ.

\section{Association of individual items of the HPRQ measure and outcomes (study objective 2)}

We explored whether certain indicators of HPRQ were more strongly associated than others with distress, treatment burden, medication convenience, self-efficacy, and satisfaction with provider by correlating each item on the HPRQ measure with each outcome measure. Relative strength of the associations was determined in 2 ways. First, we computed a mean of the correlations of each item with every outcome measure (ie, a mean of the correlation magnitudes across measures). Second, we rank-ordered the strength of the correlation magnitudes of each of the 7 HPRQ items with each individual outcome measure (ie, rank-ordering correlation magnitudes within each outcome measure), and then we computed the median rank for each item across all of the outcome measures. A higher mean correlation magnitude and a higher median rank indicate a stronger association of item with outcomes. Similar parameter-ranking procedures have been used in other studies to compare measurement properties of patient-reported outcome measures. ${ }^{36,37}$

Finally, we performed independent samples $t$-tests on the 7 HPRQ items by adherence to prescribed medications (dichotomized), and computed effect sizes $(d)$ for the group differences. Larger effect sizes are indicative of items showing a greater ability to discriminate the groups. Given that directional hypotheses are supported by prior research findings and theoretical mechanisms, ${ }^{5-8,10-12,14,15,18,35}$ a 1-tailed alpha was used and set at 0.05 for all correlations and grouplevel comparisons. However, there were no hypotheses regarding which particular HPRQ items would be most strongly associated with the outcomes. All analyses were conducted in IBM SPSS Statistics version $20^{\circledR}$.

\section{Results}

\section{Sample characteristics}

Of 838 mailed surveys, 332 were completed and returned (40\% response). In comparing survey responders with nonresponders across the stratification factors, more responders (57\%) than nonresponders (46\%) were $\geq 65$ years of age $(P<0.01)$, and fewer responders $(32 \%)$ than nonresponders (42\%) had only $1-8$ encounters with a medical provider in the last 18 months $(P<0.01)$. There were no significant differences between responders and nonresponders in the number of diagnosed conditions. Demographic, medical, and other health-related characteristics of the sample are identified in Table 1. There were more female (56\%) than male participants, with a mean age of 66 years (range: 26-90). Most (73\%) were white; however, over 20\% identified with a racial minority group, including $15 \%$ identifying as African American. Most (70\%) had at least some formal college or university education, while $27 \%$ had no more than a high school education. Among medical variables extracted from the electronic medical record, the median number of diagnosed conditions was 3 (with over one-third having $\geq 4$ conditions) and the median number of encounters with a medical provider in the past 18 months was 13.5 . The most common diagnoses were hypertension (77\%), lipid metabolic disorders $(76 \%)$, diabetes $(36 \%)$, osteoarthritis $(36 \%)$, and coronary artery disease (19\%); however, asthma, chronic kidney disease, depression, glaucoma, and COPD were also common. Sixty-three percent of participants were taking at least 4 prescribed medications, with one-third taking $\geq 6$. Thirty percent felt their physical health was fair or poor, while $23 \%$ felt their mental health was fair or 
Table I Demographic, medical, and other health-related characteristics of survey respondents $(\mathrm{N}=332)$

\begin{tabular}{|c|c|}
\hline \multicolumn{2}{|l|}{ Site (N, \%) } \\
\hline Mayo Clinic & I $78(54)$ \\
\hline Hennepin County Medical Center & $154(46)$ \\
\hline Mean age in years $(\mathrm{SD})$ & $65.9(11.0)$ \\
\hline Range & $26-90$ \\
\hline \multicolumn{2}{|l|}{ Gender (N, \%) } \\
\hline Female & $185(56)$ \\
\hline Male & $147(44)$ \\
\hline \multicolumn{2}{|l|}{ Race $(\mathrm{N}, \%)$} \\
\hline White & $24 \mid(73)$ \\
\hline Black/African-American & $5 I(I 5)$ \\
\hline Mixed & $10(3)$ \\
\hline Asian & $8(2)$ \\
\hline Native American/American Indian & $6(2)$ \\
\hline Missing & $16(5)$ \\
\hline Hispanic/Latino ethnicity (N, \%) & $11(3)$ \\
\hline \multicolumn{2}{|l|}{ Education level $(\mathrm{N}, \%)$} \\
\hline Some college/associate's degree & $119(36)$ \\
\hline College graduate (B.A., B.S., advanced degree) & $113(34)$ \\
\hline High school graduate or less & $89(27)$ \\
\hline Missing & II (3) \\
\hline \multicolumn{2}{|l|}{ No of chronic conditions ( $\mathrm{N}, \%)$} \\
\hline 2 & $103(31)$ \\
\hline 3 & $112(34)$ \\
\hline$\geq 4$ & $117(35)$ \\
\hline Median no of conditions & 3.0 \\
\hline \multicolumn{2}{|l|}{ Diagnosed chronic conditions ( $\mathrm{N}, \%)$} \\
\hline Hypertension & $254(77)$ \\
\hline Disorders of lipid metabolism (including & $252(76)$ \\
\hline \multicolumn{2}{|l|}{ hypercholesterolemia) } \\
\hline Diabetes mellitus (type I or 2) & $120(36)$ \\
\hline Osteoarthritis & $119(36)$ \\
\hline Coronary artery disease & $63(19)$ \\
\hline Asthma & $47(14)$ \\
\hline Chronic kidney disease & $44(13)$ \\
\hline Depression & $44(13)$ \\
\hline Glaucoma & $4 I(12)$ \\
\hline COPD & $34(10)$ \\
\hline Hepatitis (B and C) & $28(9)$ \\
\hline Congestive heart failure & $22(7)$ \\
\hline Rheumatoid arthritis & $15(5)$ \\
\hline Median no of provider encounters in last 18 months & 13.5 \\
\hline \multicolumn{2}{|l|}{ No of prescription medications ( $\mathrm{N}, \%)$} \\
\hline 1 & II (3) \\
\hline $2-3$ & $80(24)$ \\
\hline $4-5$ & $97(29)$ \\
\hline$\geq 6$ & III (34) \\
\hline Missing & $33(10)$ \\
\hline \multicolumn{2}{|l|}{ "How would you rate your physical health?" (N, \%) } \\
\hline Excellent & $14(4)$ \\
\hline Very good & $79(24)$ \\
\hline Good & $132(40)$ \\
\hline Fair & $69(2 I)$ \\
\hline Poor & $30(9)$ \\
\hline Missing & $8(2)$ \\
\hline \multicolumn{2}{|c|}{$\begin{array}{l}\text { "How would you rate your mental health, including your mood and } \\
\text { ability to think?" ( } \mathrm{N}, \%)\end{array}$} \\
\hline Excellent & $54(16)$ \\
\hline Very good & $109(33)$ \\
\hline
\end{tabular}

Table I (Continued)

\begin{tabular}{ll}
\hline Good & $88(27)$ \\
Fair & $59(18)$ \\
Poor & $15(5)$ \\
Missing & $7(2)$ \\
Adherence to medications in a typical week? (N, \%) & \\
Always take all medications & $260(78)$ \\
Usually/sometimes take all medications & $5 \mathrm{I}(\mathrm{I} 5)$ \\
Missing & $21(6)$ \\
Mean healthcare provider rating (SD) $(0-$ worst to & $8.7(1.7)$ \\
I0 - best) & \\
Range & $0-10$ \\
\hline
\end{tabular}

poor. Most (78\%) reported that they always take all of their medications. Overall satisfaction with healthcare providers was high (mean rating $=8.7$ out of 10 ).

\section{Association of HPRQ with outcomes (study objective I)}

Internal consistency reliability of the HPRQ measure was excellent (Cronbach's alpha $=0.96$ ). As shown in Table 2, the HPRQ measure was significantly correlated with several of the outcome measures in hypothesized directions. Higher (better) HPRQ was negatively correlated with chronic condition distress (CCDS: $r=-0.31$ ) as well as the 3 scales of the PETS treatment burden measure: Medical Information Burden $(r=-0.47)$, Difficulty with Healthcare Services $(r=-0.55)$, and Physical/Mental Exhaustion with self-management $\left(r=-0.33\right.$; all $\left.P_{\mathbf{s}}<0.001\right)$. Furthermore, higher HPRQ was positively correlated with self-reported convenience of taking required medication (TSQM Convenience: $r=0.26$ ), self-management self-efficacy (PMCSM: $r=0.33)$, and overall satisfaction with providers $(r=0.55$; all $P_{\mathrm{S}}<0.001$ ). Most of these correlations (6 of 7 or $86 \%$ ) were medium to large in magnitude. After controlling for 2 potential confounding variables, self-reported physical and mental health, all correlation magnitudes remained significantly $>0$ and were in the hypothesized directions (shown by partial correlations in the last 2 columns of Table 2). Correlation magnitudes were slightly attenuated after controlling for these variables; however, $50 \%$ (7 of 14) remained in the medium-to-large range. HPRQ was not associated with number of diagnosed conditions, number of encounters with medical providers in the past 18 months, or number of prescription medications.

The HPRQ measure differentiated respondents based on self-reported medication adherence. As hypothesized, survey respondents who reported "always" taking all of their prescribed medications $(\mathrm{n}=260)$ reported a higher HPRQ score (mean $=82.4$ ) than those who reported taking 
Table 2 Correlation $(r)$ of healthcare provider relational quality scale with other measures (zero-order and partial)

\begin{tabular}{|c|c|c|c|}
\hline Correlate measure or variable & $\begin{array}{l}\text { Healthcare } \\
\text { provider relational } \\
\text { quality }^{\mathbf{a}}\end{array}$ & $\begin{array}{l}\text { Healthcare provider } \\
\text { relational quality, controlling } \\
\text { for physical health }\end{array}$ & $\begin{array}{l}\text { Healthcare provider }{ }^{\mathrm{b}} \\
\text { relational quality, controlling } \\
\text { for mental health }\end{array}$ \\
\hline Chronic condition distress $^{c}$ & $-0.3 \mid * * *$ & $-0.26 * * *$ & $-0.20 * *$ \\
\hline PETS Medical Information Burden ${ }^{d}$ & $-0.47 * * *$ & $-0.42 * * *$ & $-0.36 * * *$ \\
\hline PETS Difficulty with Healthcare Services ${ }^{d}$ & $-0.55^{* * *}$ & $-0.55^{* * *}$ & $-0.5 I^{* * *}$ \\
\hline PETS Physical/Mental Exhaustion ${ }^{d}$ & $-0.33^{* * *}$ & $-0.34 * * *$ & $-0.25 * * *$ \\
\hline TSQM Medication Convenience $^{e}$ & $0.26 * * *$ & $0.20 * *$ & $0.14^{*}$ \\
\hline PMCSM (self-efficacy) ${ }^{\dagger}$ & $0.33 * * *$ & $0.23 * * *$ & $0.16 * *$ \\
\hline Health care provider rating ( 0 - worst to 10 - best $)$ & $0.55^{* * *}$ & $0.59 * * *$ & $0.58 * * *$ \\
\hline No of chronic conditions & -0.02 & 0.01 & 0.01 \\
\hline No of provider encounters (last 18 months) & -0.05 & -0.02 & -0.03 \\
\hline No of prescription medications & -0.01 & 0.06 & 0.06 \\
\hline
\end{tabular}

Notes: ${ }^{a}$ Zero-order (bivariate) correlations. ${ }^{b}$ Partial correlations controlling for the indicated variable. 'Higher score indicates greater distress. ${ }^{\mathrm{d}} \mathrm{PETS}$ : higher scores indicate greater treatment burden. ${ }^{\mathrm{e}} \mathrm{TSQM}$ : medication convenience scale, higher score indicates greater medication convenience. ${ }^{\mathrm{f} P M C S M: ~ h i g h e r ~ s c o r e ~ i n d i c a t e s ~ g r e a t e r ~ s e l f-e f f i c a c y ~}$ for self-management. ${ }^{*} P<0.05$ (I-tailed); ${ }^{*} * P<0.0$ I (I-tailed); ${ }^{*} * P<0.00 \mathrm{I}$ (I-tailed).

Abbreviations: PETS, Patient Experience with Treatment and Self-management; TSQM, Treatment Satisfaction Questionnaire for Medication; PMCSM, Perceived Medical Condition Self-management.

all of their medications "usually" or "sometimes" $(\mathrm{n}=51)$ (mean $=70.4 ; t$ [294] $=3.79, P<0.001$; Cohen's $d=0.59$, a medium-to-large effect size).

\section{Association of individual HPRQ items with outcomes (study objective 2)}

As shown in Table 3, all HPRQ scale items were significantly associated with all outcome measures (all $P_{\mathrm{S}}<0.001$ ) with over half of the correlations ( 29 of 49 or $59 \%$ ) in the mediumto-large range of magnitude (ie, $r \geq 0.30$ ). A summary of the relative strengths of the associations between items and outcomes is indicated in the last column of the table. This column features the following: 1) the mean of the correlation magnitudes for each item across all outcomes with a higher mean magnitude indicating stronger association with outcomes, and 2) the median rank of the rank-ordered correlation magnitudes of each HPRQ item and each individual outcome measure, with a higher median rank indicating a stronger association of item with outcomes (note: a higher median rank is indicated by a lower number). As shown by the summary statistics in the last column of Table 3, the HPRQ items showing the strongest associations with outcomes were "I have trust in my healthcare provider(s)" (mean $r=0.39$, median rank $=2$ ), "My healthcare provider(s) spends enough time with me" (mean $r=0.38$, median rank $=2$ ), and "My healthcare provider(s) listens carefully to me" (mean $r=0.36$, median rank $=3$ ). All of the mean correlations were just above a medium magnitude.

Finally, independent samples $t$-tests were conducted on each of the 7 HPRQ items by self-reported adherence to prescribed medications ("always" vs "usually/sometimes") to determine if certain items were better at discriminating groups. Comparisons of item discriminability were made by comparing effect sizes $(d)$ corresponding to mean differences in item scores between groups, with larger effect sizes indicating greater ability of the item to discriminate. As shown in Figure 1, all 7 HPRQ items discriminated the groups. Respondents who reported "always" taking all of their prescribed medications reported better HPRQ in all aspects than those who reported taking all of their medications "usually" or "sometimes" (all $P_{\mathrm{s}}<0.05$, effect sizes: 0.37-0.68). Three items discriminated the groups best. Medium-to-large effect sizes $^{34}$ were observed for "My healthcare provider(s) spends enough time with me" ( $d=0.68)$, "My provider(s) listens carefully to me" $(d=0.62)$, and "I have trust in my healthcare provider(s)" $(d=0.60)$.

\section{Discussion}

Our study showed that the quality of the patient-provider relationship, as perceived by patients, is associated with self-management, treatment burden, psychosocial outcomes, and satisfaction with providers in people coping with MCCs. Patients who believed that their healthcare providers exhibited good communication and interpersonal skills and treated them with dignity and respect perceived less treatment and self-management burden, reported less distress, felt greater confidence in their ability to self-manage, and were more satisfied with their providers. These relationships remained after controlling for physical and mental health status, and were mostly medium-to-large in magnitude. Furthermore, patients who reported being $100 \%$ adherent to prescribed medications perceived better HPRQ than patients who reported less than 


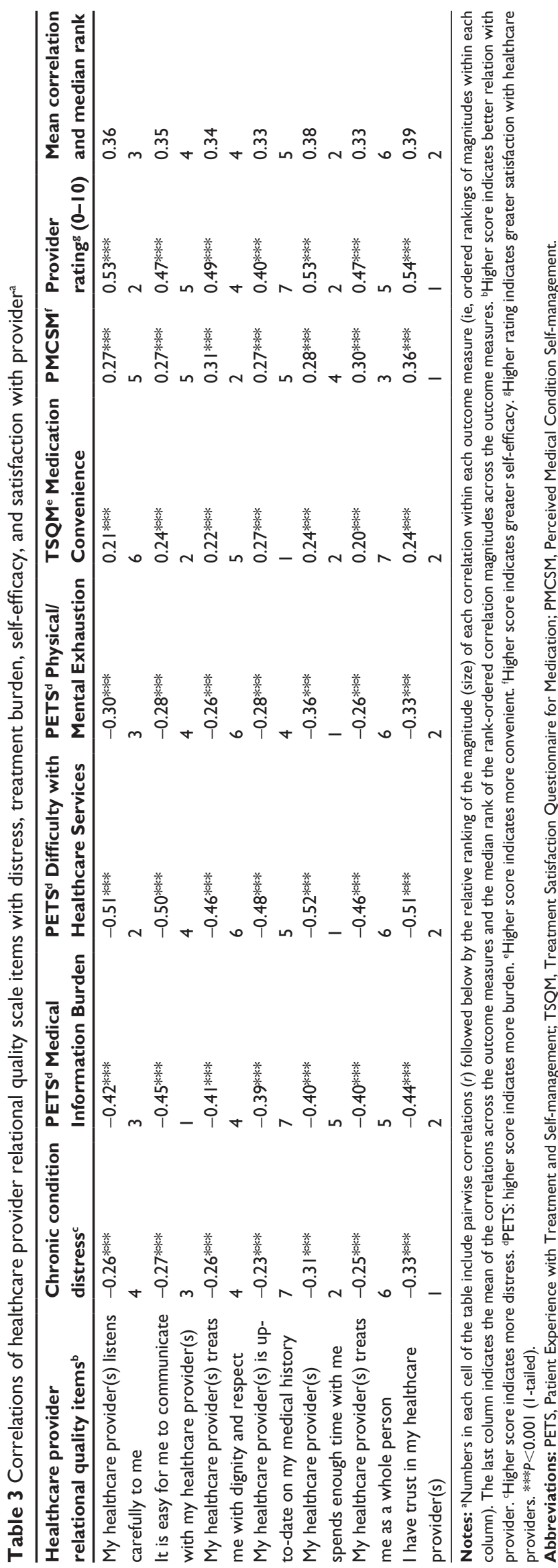

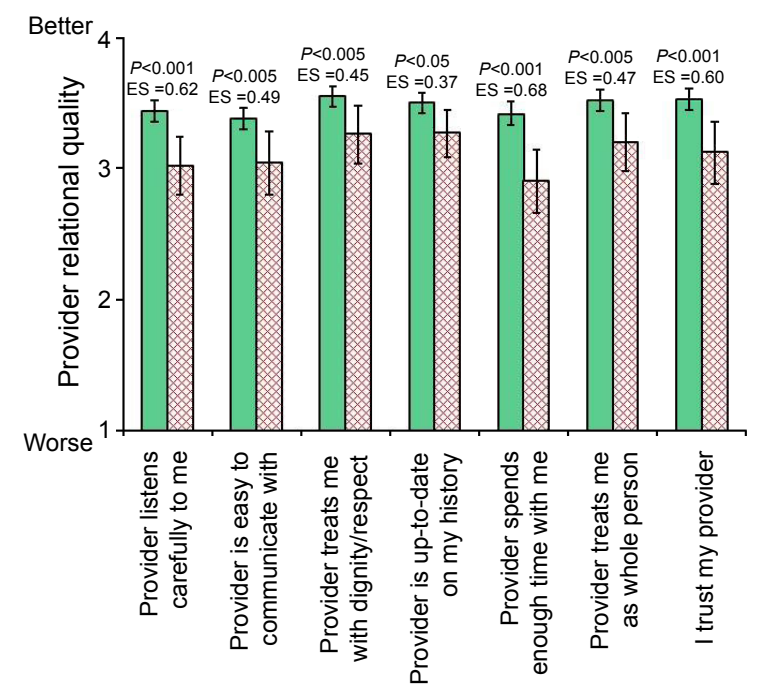

$\square$ Always take all Usually or sometimes

medications take all medications

Figure I Mean healthcare provider relational quality items by patient-reported medication adherence with $95 \%$ confidence intervals.

Abbreviation: ES, effect size.

perfect adherence. HPRQ was not associated with objective indicators such as number of diagnosed conditions, number of recent encounters with providers, or the number of medications being taken. While it is tempting to speculate that having a better relationship with one's provider might lead to better outcomes, the cross-sectional design of our study precludes drawing causal conclusions. Prospective studies are needed to further specify and test the pathways through which HPRQ operates. ${ }^{12}$ However, our findings are consistent with those of other studies and meta-analytic reviews that have shown elements of HPRQ to be associated with less distress and perceived treatment burden, ${ }^{11,18}$ higher self-efficacy, ${ }^{10}$ better self-management ability, ${ }^{6}$ greater adherence to treatment, ${ }^{8,11}$ and satisfaction with healthcare providers. ${ }^{35}$

We also disaggregated HPRQ to understand the relationship between each of its constituent elements and outcomes. The HPRQ items reflecting that the healthcare provider spends enough time with the patient, listens carefully to the patient, and is trusted by the patient featured the strongest associations with the outcomes, with correlation magnitudes and effect sizes in the medium-to-large range. These items were also most strongly correlated with overall satisfaction with the provider. A recent study by Stucky et a ${ }^{38}$ has shown that an aggregate scale of 2 CG-CAHPS items, "spending enough time" with the patient and "listening carefully" to the patient, provides nearly equivalent measurement precision as the longer Doctor Communication composite scale in which they are embedded. The aggregate scale maintained the 
validity of the longer composite, with scores remaining highly correlated with overall satisfaction with the provider. ${ }^{38}$ Our study confirms use of these 2 items to assess HPRQ.

Our study also supports addition of an overall rating of patient trust in the provider to the measurement of HPRQ. We observed robust associations of this item with patient outcomes, with most correlation magnitudes and effect sizes in the medium-to-large range. Trust is a global impression, an attitude, formed about the provider and stemming from a patient's belief that the provider is not only technically competent but also honest, empathic, and concerned about the patient's welfare and best interests. ${ }^{15,39}$ Measuring trust is less emphasized in standard patient-reported experience measures vis-à-vis more specific, tangible elements of care quality such as providing information. ${ }^{13}$ However, with evidence linking patient trust in the provider to treatment adherence and care continuity, ${ }^{14,15}$ more consistent measurement of it appears warranted. In primary care, there have been recent calls to employ more patient-centric measures as quality indicators, including patient experience measures that tap into feelings of trust and respect with providers. ${ }^{40}$ Organizational commitment to both measuring patient trust and supporting providers to pursue it could foster a health care climate where empathic care is considered normative.

\section{Practical implications: teaching relational skills to healthcare providers}

While medical education has paid somewhat more attention to improving communication through optimizing information provision, several studies indicate that interpersonal skills can also be effectively taught to physicians. ${ }^{41-43}$ A 2009 metaanalysis concluded that physician training in communication skills, including both task-oriented (eg, information provision) and psychosocial skills (eg, rapport and trust building), can result in substantial improvements in patient adherence to treatment. ${ }^{8}$ Further evidence suggests that building an atmosphere of trust and nonjudgmental acceptance within the medical encounter may facilitate more open and honest discussions between providers and patients about self-care. ${ }^{19}$ While many studies have focused on training physicians, enhancing communication and relational skills in other healthcare providers could have important implications as well. Studies have shown that patients are more adherent to treatment plans and more satisfied with their care when they interact with nurse practitioners who have a "biopsychosocial" style of communication - communication that actively engages patients in decisions about their care and takes into account their socioemotional environment. ${ }^{44}$ Furthermore, communication skills training may be useful for clinical pharmacists. Recent evidence suggests that clinical pharmacists focus largely on biomedical issues in their encounters with patients and pay little attention to psychosocial issues, including those that may affect medication adherence. ${ }^{45,46}$ Given the importance of multidisciplinary care teams in the management of patients with chronic illnesses, ${ }^{47}$ optimal patient outcomes are more likely to be realized when all members of the clinical care team are proficient in patient-centered communication.

\section{Limitations}

First, our study's cross-sectional design precludes drawing causal conclusions about the effects of HPRQ. It is possible that patients more adept at self-management and lower in distress are simply easier for providers to relate to and work with, leading to more favorable impressions by the patients. Second, most of the data for this analysis relied on patient self-reports. More objective outcomes extracted from patient medical records or administrative claims could be used in future studies to further clarify the role of HPRQ in patient outcome. In particular, self-reporting of medication adherence is more accurately construed as assessing perceived, rather than actual adherence. The single-item measure used in this study lacks comprehensiveness ${ }^{48}$ and may be subject to social desirability bias (ie, skewed toward greater reporting of adherence). Hence, actual group differences in HPRQ across medication adherence may be larger than what was observed in this study. Socially desirable responding in the HPRQ measure (ie, toward higher rating of provider relational quality) could also have attenuated some of the correlations with the other outcome measures due to restricted range in the scores. Third, our measure of HPRQ was intentionally brief and reflected issues voiced in interviews and focus groups of a select sample of patients with MCCs. As the parent study was focused on deriving and testing a multi-domain measure of treatment and self-management burden, ${ }^{3}$ we were less interested in simultaneously developing a comprehensive measure of HPRQ. Hence, there may be some important aspects of this concept that are missing from our scale. Still, we believe that valuable lessons can be learned from even very brief measures of potential mitigating influences of treatment and self-management burden. Future research that compares various resources that patients may draw on to relieve burden or that investigates the impact of multiple accumulated resources is rendered more feasible when measurements of diverse constructs are targeted and brief. Finally, as the HPRQ scale did not specify a particular provider or provider type, we do not know which provider 
(or providers) the patient was referencing when rating the items. We only know that they were thinking of those health professionals who were currently providing them with medical care for their health conditions.

\section{Conclusion}

Our study augments the growing body of evidence that relational skills of healthcare providers may influence the ability of chronically ill patients to self-manage their conditions. Not only do good communication and interpersonal skills of providers appear to be important, but so too does the degree of trust that patients have in their providers. Our data suggest that a simple assessment of patient trust could augment traditional patient experience measures of the patient-provider relationship. Future research should prospectively test the influence of HPRQ on self-management outcomes like treatment burden, as well as compare the effects of HPRQ to other resources that patients may draw on to help manage their health (eg, individual coping ability, support from caregivers, etc). Our findings and those of others indicate that the quality of the patient-provider relationship does seem to matter. Future investigation should focus on the extent to which it does.

\section{Acknowledgments}

The research reported in this manuscript was supported by the National Institute of Nursing Research of the National Institutes of Health (USA) under award number R21NR012984. The content is solely the responsibility of the authors and does not necessarily represent the official views of the National Institutes of Health. The authors thank Ms Ann Harris and personnel in the Mayo Clinic Survey Research Center for formatting, distribution, and receipt of the survey battery.

\section{Disclosure}

The authors report no conflicts of interest in this work.

\section{References}

1. Cramm JM, Nieboer AP. Self-management abilities, physical health and depressive symptoms among patients with cardiovascular diseases, chronic obstructive pulmonary disease, and diabetes. Patient Educ Couns. 2012;87(3):411-415.

2. Boyd CM, Wolff JL, Giovannetti E, et al. Healthcare task difficulty among older adults with multimorbidity. Med Care. 2014;52 Suppl 3: S118-S125.

3. Eton DT, Yost KJ, Lai JS, et al. Development and validation of the Patient Experience with Treatment and Self-management (PETS): a patientreported measure of treatment burden. Qual Life Res. 2017;26(2): 489-503.

4. Ridgeway JL, Egginton JS, Tiedje K, et al. Factors that lessen the burden of treatment in complex patients with chronic conditions: a qualitative study. Patient Prefer Adherence. 2014;8:339-351.
5. Anhang Price R, Elliott MN, Zaslavsky AM, et al. Examining the role of patient experience surveys in measuring health care quality. Med Care Res Rev. 2014;71(5):522-554.

6. Cramm JM, Nieboer AP. Chronically ill patients' self-management abilities to maintain overall well-being: what is needed to take the next step in the primary care setting? BMC Fam Pract. 2015;16:123.

7. White RO, Eden S, Wallston KA, et al. Health communication, selfcare, and treatment satisfaction among low-income diabetes patients in a public health setting. Patient Educ Couns. 2015;98(2):144-149.

8. Zolnierek KB, Dimatteo MR. Physician communication and patient adherence to treatment: a meta-analysis. Med Care. 2009;47(8): 826-834.

9. Street RL Jr, Elwyn G, Epstein RM. Patient preferences and healthcare outcomes: an ecological perspective. Expert Rev Pharmacoecon Outcomes Res. 2012;12(2):167-180.

10. Finney Rutten LJ, Hesse BW, St Sauver JL, et al. Health self-efficacy among populations with multiple chronic conditions: the value of patient-centered communication. Adv Ther. 2016;33(8):1440-1451.

11. Linetzky B, Jiang D, Funnell MM, Curtis BH, Polonsky WH. Exploring the role of the patient-physician relationship on insulin adherence and clinical outcomes in type 2 diabetes: insights from the MOSAIc study. J Diabetes. 2017;9(6):596-605.

12. Street RL Jr, Makoul G, Arora NK, Epstein RM. How does communication heal? Pathways linking clinician-patient communication to health outcomes. Patient Educ Couns. 2009;74(3):295-301.

13. Quigley DD, Martino SC, Brown JA, Hays RD. Evaluating the content of the communication items in the CAHPS clinician and group survey and supplemental items with what high-performing physicians say they do. Patient. 2013;6(3):169-177.

14. Schoenthaler A, Montague E, Baier Manwell L, Brown R, Schwartz MD, Linzer M. Patient-physician racial/ethnic concordance and blood pressure control: the role of trust and medication adherence. Ethn Health. 2014;19(5):565-578.

15. Thom DH, Hall MA, Pawlson LG. Measuring patients' trust in physicians when assessing quality of care. Health Aff (Millwood). 2004; 23(4):124-132.

16. Safran DG, Taira DA, Rogers WH, Kosinski M, Ware JE, Tarlov AR. Linking primary care performance to outcomes of care. J Fam Pract. 1998;47(3):213-220.

17. Fox S, Chesla C. Living with chronic illness: a phenomenological study of the health effects of the patient-provider relationship. J Am Acad Nurse Pract. 2008;20(3):109-117.

18. Mohammed MA, Moles RJ, Chen TF. Medication-related burden and patients' lived experience with medicine: a systematic review and metasynthesis of qualitative studies. BMJ Open. 2016;6(2):e10035.

19. Ritholz MD, Beverly EA, Brooks KM, Abrahamson MJ, Weinger K. Barriers and facilitators to self-care communication during medical appointments in the United States for adults with type 2 diabetes. Chronic Illn. 2014;10(4):303-313.

20. Hall JA, Roter DL, Katz NR. Task versus socioemotional behaviors in physicians. Med Care. 1987;25(5):399-412.

21. Stewart AL, Napoles-Springer AM, Gregorich SE, Santoyo-Olsson J. Interpersonal processes of care survey: patient-reported measures for diverse groups. Health Serv Res. 2007;42(3 Pt 1):1235-1256.

22. Jenkinson C, Coulter A, Bruster S. The Picker Patient Experience Questionnaire: development and validation using data from in-patient surveys in five countries. Int J Qual Health Care. 2002;14(5):353-358.

23. Dyer N, Sorra JS, Smith SA, Cleary PD, Hays RD. Psychometric properties of the Consumer Assessment of Healthcare Providers and Systems $\left(\right.$ CAHPS $\left.^{\circledR}\right)$ Clinician and Group Adult Visit Survey. Med Care. 2012; 50 Suppl:S28-S34.

24. Anderson LA, Dedrick RF. Development of the Trust in Physician scale: a measure to assess interpersonal trust in patient-physician relationships. Psychol Rep. 1990;67(3 Pt 2):1091-1100.

25. Lee PG, Cigolle C, Blaum C. The co-occurrence of chronic diseases and geriatric syndromes: the health and retirement study. J Am Geriatr Soc. 2009;57(3):511-516. 
26. Schneider KM, O’Donnell BE, Dean D. Prevalence of multiple chronic conditions in the United States' Medicare population. Health Qual Life Outcomes. 2009;7:82.

27. Schoenberg NE, Leach C, Edwards W. "It's a toss up between my hearing, my heart, and my hip": prioritizing and accommodating multiple morbidities by vulnerable older adults. J Health Care Poor Underserved. 2009;20(1):134-151.

28. Vogeli C, Shields AE, Lee TA, et al. Multiple chronic conditions: prevalence, health consequences, and implications for quality, care management, and costs. J Gen Intern Med. 2007;22(Suppl 3):391-395.

29. Cella D. Manual of the Functional Assessment of Chronic Illness Therapy (FACIT) Measurement System - Version 4. Evanston, IL: Center on Outcomes, Research \& Education (CORE), Evanston Northwestern Healthcare and Northwestern University; 1997.

30. Atkinson MJ, Sinha A, Hass SL, et al. Validation of a general measure of treatment satisfaction, the Treatment Satisfaction Questionnaire for Medication (TSQM), using a national panel study of chronic disease. Health Qual Life Outcomes. 2004;2:12.

31. Polonsky WH, Fisher L, Earles J, et al. Assessing psychosocial distress in diabetes: development of the Diabetes Distress Scale. Diabetes Care. 2005;28(3):626-631.

32. Wallston KA, Osborn CY, Wagner LJ, Hilker KA. The Perceived Medical Condition Self-Management Scale applied to persons with HIV/AIDS. J Health Psychol. 2011;16(1):109-115.

33. Hays RD, Bjorner JB, Revicki DA, Spritzer KL, Cella D. Development of physical and mental health summary scores from the patient-reported outcomes measurement information system (PROMIS) global items. Qual Life Res. 2009;18(7):873-880.

34. Cohen J. Statistical Power Analysis for the Behavioral Sciences. 2nd ed. Hillsdale, NJ: Lawrence Erlbaum Associates; 1988.

35. Hall JA, Roter DL, Katz NR. Meta-analysis of correlates of provider behavior in medical encounters. Med Care. 1988;26(7):657-675.

36. Eurich DT, Johnson JA, Reid KJ, Spertus JA. Assessing responsiveness of generic and specific health related quality of life measures in heart failure. Health Qual Life Outcomes. 2006;4:89.

37. Birbeck GL, Kim S, Hays RD, Vickrey BG. Quality of life measures in epilepsy: how well can they detect change over time? Neurology. 2000;54(9):1822-1827.
38. Stucky BD, Hays RD, Edelen MO, Gurvey J, Brown JA. Possibilities for shortening the CAHPS Clinician and Group Survey. Med Care. 2016;54(1):32-37.

39. Carter MA. Trust, power, and vulnerability: a discourse on helping in nursing. Nurs Clin North Am. 2009;44(4):393-405.

40. Young RA, Roberts RG, Holden RJ. The challenges of measuring, improving, and reporting quality in primary care. Ann Fam Med. 2017; 15(2):175-182.

41. Branch WT Jr, Frankel R, Gracey CF, et al. A good clinician and a caring person: longitudinal faculty development and the enhancement of the human dimensions of care. Acad Med. 2009;84(1):117-125.

42. Dyche L. Interpersonal skill in medicine: the essential partner of verbal communication. J Gen Intern Med. 2007;22(7):1035-1039.

43. Meyer EC, Sellers DE, Browning DM, McGuffie K, Solomon MZ, Truog RD. Difficult conversations: improving communication skills and relational abilities in health care. Pediatr Crit Care Med. 2009; 10(3):352-359.

44. Charlton CR, Dearing KS, Berry JA, Johnson MJ. Nurse practitioners' communication styles and their impact on patient outcomes: an integrated literature review. J Am Acad Nurse Pract. 2008;20(7):382-388.

45. Murad MS, Chatterley T, Guirguis LM. A meta-narrative review of recorded patient-pharmacist interactions: exploring biomedical or patient-centered communication? Res Social Adm Pharm. 2014; 10(1):1-20.

46. Driesenaar JA, De Smet PA, van Hulten R, Hu L, van Dulmen S. Communication during counseling sessions about inhaled corticosteroids at the community pharmacy. Patient Prefer Adherence. 2016;10: 2239-2254.

47. Wagner EH. The role of patient care teams in chronic disease management. BMJ. 2000;320(7234):569-572.

48. Lavsa SM, Holzworth A, Ansani NT. Selection of a validated scale for measuring medication adherence. J Am Pharm Assoc (2003). 2011; 51(1):90-94 


\section{Supplementary material}

Table SI Medical Information Burden, Difficulty with Healthcare Services, and Physical/Mental Exhaustion due to self-management scales from the Patient Experience with Treatment and Self-management

\section{Medical Information Burden}

In the past 4 weeks, how easy/difficult has it been to...

- Learn about your health problem(s)?

- Learn what foods you should eat to stay healthy?

- Find information on the medications that you have to take?

- Understand changes to your treatment plan?

- Understand the reasons why you are taking some medicines?

- Find sources of medical information that you trust?

- Understand advice from different healthcare providers?

Responses: very easy, easy, neither easy nor difficulty, difficult, very difficult, not applicable

Difficulty with Healthcare Services

Thinking about your health care, how much do you agree/disagree with the following?

- I have problems with different healthcare providers not communicating with each other about my medical care

- I have to see too many different specialists for my health problem(s) or illness(es)

- I have problems filling out forms related to my health care

- I have problems getting appointments at times that are convenient for me

- I have problems getting appointments with a specialist

- I have to wait too long at my medical appointments

- I have to wait too long at the pharmacy for my medicine

Responses: strongly agree, agree, disagree, strongly disagree, not applicable

Physical/Mental Exhaustion due to self-management

In the past 4 weeks, how often did your self-care make you feel...

- Angry?

- Preoccupied?

- Depressed?

- Worn out?

- Frustrated?

Responses: never, rarely, sometimes, often, always

\section{Publish your work in this journal}

Patient Preference and Adherence is an international, peer-reviewed, open access journal that focuses on the growing importance of patient preference and adherence throughout the therapeutic continuum. Patient satisfaction, acceptability, quality of life, compliance, persistence and their role in developing new therapeutic modalities and compounds to optimize clinical outcomes for existing disease states are major areas of interest for the journal. This journal has been accepted for indexing on PubMed Central. The manuscript management system is completely online and includes a very quick and fair peer-review system, which is all easy to use. Visit http://www. dovepress.com/testimonials.php to read real quotes from published authors.

Submit your manuscript here: http://www.dovepress.com/patient-preference-and-adherence-journal 